\title{
Integrated research efforts are needed to better understand how to reduce the proportion of patients with cancer who are diagnosed as emergencies
}

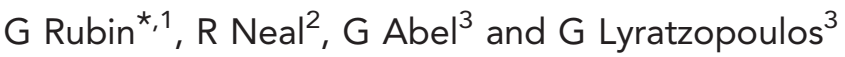 \\ ${ }^{1}$ RCGP/CRUK Clinical Lead for Cancer, Wolfson Research Institute, Queen's Campus, Durham University, Stockton on Tees TS17 \\ $6 \mathrm{BH}$, UK; ${ }^{2}$ North Wales Centre for Primary Care Research, College of Health and Behavioural Sciences Gwenfro 5, Wrexham \\ Technology Park, Wrexham LL13 7YP, UK and ${ }^{3}$ Cambridge Centre for Health Services Research, Department of Public Health and \\ Primary Care, University of Cambridge, Cambridge CB2 OSR, UK
}

Sir,

Elliss-Brookes et al (2012) provide further evidence that the challenges of achieving a prompt diagnosis of cancer vary substantially for patients with different cancers. Overall patterns of variation by cancer in the proportion of patients who are diagnosed as emergencies reported by the 'Routes-to-Diagnosis' project closely mirror data on the proportion of patients who were referred to secondary care as emergencies from the National Audit of Cancer Diagnosis in Primary Care (NACDPC), an audit of nearly 19000 patients diagnosed in 2009/10 that used general practice records as the primary data source (Rubin et al, 2011). For example, patients with breast cancer, melanoma and endometrial cancer had the lowest proportion of emergency route/referral in either publication; the opposite was true for patients with brain, lung and pancreatic cancer and multiple myeloma (Table 1). Comparing data from both publications on the proportion of patients with an emergency diagnosis (route/referral) for different cancers indicate a Spearman rank correlation coefficient of 0.97 (i.e., a very high degree of rank agreement). Similar observations can be made about patterns of variation in the proportion of unplanned first-time hospital admission for patients with different cancers (Bottle et al, 2012). These comparisons, however, also reveal substantial differences in the absolute proportions of cancer patients diagnosed as emergencies using either secondary or primary care data sources. These may reflect both differences in the studied populations, and in outcome definitions and methods used for data collection. Although the population of patients studied by the NACDCPC project adequately reflects cancer incidence statistics (Rubin et al, 2011), general practice records can be considered to be less likely to identify patients with cancer who first present as emergencies and either died during the spell of the respective hospital admission or who are transferred to a new address on discharge (e.g., as in the case of patients discharged to hospices from acute care). Similar patterns have been observed for patients with cardiovascular disease where estimates of incidence and case fatality made from hospital records are higher than GP records (Payne et al, 2012). More importantly, some patients with an emergency route to diagnosis would have no prior consultation with a general practitioner (or would see a general practitioner only once, at a phase of their illness that warrants an emergency hospital referral).

Better understanding how emergency diagnosis of cancer relates to previous encounters with primary care, and how patterns of prior consultation experience vary between different cancers, is critical to help inform the development of appropriate improvement interventions and policy initiatives. Awareness interventions might help to reduce patient delay in seeking a medical assessment, but interventions to improve the sensitivity of the appraisal of cancer symptoms by general practitioners will also be required in order to achieve a reduction in the proportion of emergency diagnoses (Lyratzopoulos et al, 2012). More detailed understanding of the inter-relationships between patient behaviour, general 
Table 1. Percentage of cancer patients who present as emergencies, by source publication (Routes; NACPC)

$\%$ Of cancer patients with emergency route to diagnosis or emergency referral

\begin{tabular}{|c|c|c|}
\hline Cancer $^{a}$ & $\begin{array}{c}\text { Routes } \\
\text { project (1) }\end{array}$ & NADCPC (2) \\
\hline Central nervous system/brain & 62 & 39 \\
\hline Pancreatic & 50 & 29 \\
\hline Lung & 39 & 20 \\
\hline Multiple myeloma & 37 & 28 \\
\hline Stomach & 33 & 21 \\
\hline Ovarian & 32 & 23 \\
\hline $\begin{array}{l}\text { Non-Hodgkin's lymphoma/ } \\
\text { lymphoma }\end{array}$ & 27 & 18 \\
\hline Colorectal & 26 & 15 \\
\hline $\begin{array}{l}\text { Renal and unspecified urinary } \\
\text { organs/renal }\end{array}$ & 25 & 13 \\
\hline Oesophageal & 22 & 10 \\
\hline Bladder & 19 & 8 \\
\hline Prostate & 10 & 7 \\
\hline Uterine/endometrial & 8 & 6 \\
\hline Breast & 5 & 4 \\
\hline Melanoma & 3 & 5 \\
\hline
\end{tabular}

a Where the term used for a specific neoplasm is different between the two publications, the term used in the Routes project appears first, followed by the corresponding term used in the NACDPC publication. These differences indicate some differences in definitions or respective sites between the two data sets, for example, lymphoma (NACDPD) as opposed to specific restriction to non-Hodgkin's lymphoma (Routes). practitioner assessment, including use of diagnostics, and routes to diagnosis is needed. These questions should be addressed by future research efforts encompassing nationwide analysis of both primary care records and routine hospital statistics.

\section{REFERENCES}

Bottle A, Tsang C, Parsons C, Majeed A, Soljak M, Aylin P (2012) Association between patient and general practice characteristics and unplanned first-time admissions for cancer: observational study. $\mathrm{Br} J$ Cancer 107(8): 1213-1219.

Elliss-Brookes L, McPhail S, Ives A, Greenslade M, Shelton J, Hiom S, Richards M (2012) Routes to diagnosis for cancer-determining the patient journey using multiple routine data sets. Br J Cancer 107(8): $1220-1226$.

Lyratzopoulos G, Greenberg DC, Rubin GP, Abel GA, Walter FM, Neal RD (2012) Advanced stage diagnosis of cancer: who is at greater risk? Expert Rev Anticancer Ther 12(8): 993-996.

Payne RA, Abel GA, Simpson CR (2012) A retrospective cohort study assessing patient characteristics and the incidence of cardiovascular disease using linked routine primary and secondary care data. $B M J$ Open 2(2): e000723.

Rubin G, McPhail S, Elliott K. National Audit of Cancer Diagnosis in Primary Care. Royal College of General Practitioners: London, England. (2011) . 